LAPTH-068/13

\title{
Bertotti-Robinson solutions in five-dimensional quadratic gravity
}

\author{
Gérard Clément* \\ LAPTh, Université de Savoie, CNRS, 9 chemin de Bellevue, \\ BP 110, F-74941 Annecy-le-Vieux cedex, France
}

December 14, 2013

\begin{abstract}
We construct new solutions of five-dimensional quadratic gravity as direct products of a constant curvature two-surface with a solution of three-dimensional new massive gravity with constant scalar curvature. These solutions could represent near-horizon limits of five-dimensional asymptotically flat black strings or black rings. A number of these non-asymptotically flat solutions are themselves black strings or rings. A by-product of our analysis is the construction of new solutions of four-dimensional quadratic gravity obtained by toroidal reduction of the five-dimensional solutions with flat transverse space. These again include black strings or rings, and an $A d S_{2} \times T^{2}$ solution of $f(R)$ gravity for a specific relation between the model parameters.
\end{abstract}

\footnotetext{
*Email:gerard.clement@lapth.cnrs.fr
} 


\section{Introduction}

It is well known that Einstein's theory of gravity does not lead upon quantization to a perturbatively renormalizable theory. The addition to the linear Einstein-Hilbert action of curvature squared terms leads to a renormalizable theory [1]. This theory is generically non-unitary due to the occurreence in the linearized theory of massive spin-2 ghost modes, which are absent only if the quadratic action is of the Einstein-Gauss-Bonnet form [2]. However, it has recently been shown that in four [3] and in higher dimensions [4] the massive spin-2 mode may be rendered massless by a (more general) suitable choice of the model parameters.

Few exact solutions of the generic theory of $D$-dimensional quadratic gravity are known, with the exception of classes of algebraically special solutions, such as AdS waves [5], and type III and type N solutions [6] (for a review and references, see [7]). This paper is devoted to the search for exact solutions of five- (and four-) dimensional quadratic gravity describing spacetimes which are the direct product of two manifolds, one of which at least has constant curvature.

The first example of such a solution is due to Nariai [8], who showed that the four-dimensional Einstein equations with a positive cosmological constant admit a solution with the geometry $d S_{2} \times S^{2}$. This has been shown to be the near-extreme, near-horizon limit of the de Sitter-Schwarzschild solution [9]. The anti-Nariai solution of the Einstein equations with a negative cosmological constant, with the geometry $A d S_{2} \times H^{2}$, can be generated from the Nariai solution by an appropriate duality transformation [10. Better known is the Bertotti-Robinson solution [11] of four-dimensional EinsteinMaxwell theory, representing a spacetime with the geometry $A d S_{2} \times S^{2}$ supported by a monopole electric or magnetic flux. This is the near-horizon limit of the extreme Reissner-Nordström black hole, and can be generalized to a solution of the Einstein-Maxwell equations with cosmological constant [11] (for a review, see [12]).

The anti-Nariai and Bertotti-Robinson solutions have been generalized to solutions of the $D$-dimensional cosmological Einstein equations with the geometry $A d S_{2} \times \Sigma_{D-2}$, with $\Sigma_{D-2}$ a constant curvature Riemannian manifold or a product of such manifolds [13, 14, 15, 16]. These are the near-horizon limits of $D$-dimensional black holes. Near-horizon geometries of extremal black hole solutions to higher-dimensional supergravities were classified in [17. It has been shown that such product spacetimes also solve the $D$ dimensional Einstein-Gauss-Bonnet equations [18] and, more generally, the $D$-dimensional Einstein-Maxwell-dilaton equations with all possible higher 
order corrections [19]. In this paper, we shall consider a different generalization to $D=5$ of the Bertotti-Robinson solution, namely product spacetimes with the geometry $M_{3} \times \Sigma_{2}$, where $M_{3}$ is a (not necessarily constant curvature) Lorentzian manifold, and $\Sigma_{2}=S^{2}, T^{2}$ or $H^{2}$ is constant curvature. These could represent near-horizon limits of five-dimensional black strings or black rings.

To construct such solutions to five-dimensional quadratic gravity, we shall follow the strategy initiated in [20]. The direct product ansatz splits the five-dimensional field equations into a longitudinal set of three-dimensional field equations (for the $M_{3}$ ), and a transverse set of two-dimensional field equations (for the $\Sigma_{2}$ ), which in the case of a constant curvature $\Sigma_{2}$ amount to a constraint on the curvature invariants of the $M_{3}$. This reduction is carried out in Section 2. It turns out that in the present case the threedimensional field equations are those of new massive gravity (NMG) [21, while the constraint equation can be solved for a constant scalar curvature. In Section 3 we review the various known solutions of NMG with constant scalar curvature, and the resulting uplifted solutions to five-dimensional quadratic gravity. Depending on the values of the coupling constants, these include in particular black strings and black rings. In the special case of a flat transverse space $\Sigma_{2}$, these five-dimensional solutions can be toroidally reduced to a class of non-asymptotically flat cylindrical solutions of fourdimensional quadratic gravity with the geometry $M_{3} \times S^{1}$, again including some black strings or black rings. Our results are summarized in the last section.

\section{Reduction of five-dimensional quadratic gravity}

The generic action for $D$-dimensional quadratic gravity is 22$]$

$I_{(D)}=\int \mathrm{d}^{D} x \sqrt{\left|g_{(D)}\right|}\left\{\frac{\mathcal{R}-2 \Lambda}{\kappa}+\alpha \mathcal{R}^{2}+\beta \mathcal{R}^{\mu \nu} R_{\mu \nu}+\gamma\left(\mathcal{R}^{\mu \nu \rho \sigma} \mathcal{R}_{\mu \nu \rho \sigma}-4 \mathcal{R}^{\mu \nu} \mathcal{R}_{\mu \nu}+\mathcal{R}^{2}\right)\right\}$.

where $\kappa$ is the $D$-dimensional Einstein constant, $\Lambda$ the cosmological constant and $\alpha, \beta, \gamma$ the quadratic coupling constants.

The equations of motion that follow from (2.1) are

$$
\begin{aligned}
& \frac{1}{\kappa}\left(\mathcal{R}_{\mu \nu}-\frac{1}{2} g_{(D) \mu \nu} \mathcal{R}+\Lambda g_{(D) \mu \nu}\right)+2 \alpha \mathcal{R}\left(\mathcal{R}_{\mu \nu}-\frac{1}{4} g_{(D) \mu \nu} \mathcal{R}\right) \\
& +2 \beta\left(\mathcal{R}_{\mu \sigma \nu \rho}-\frac{1}{4} g_{(5) \mu \nu} \mathcal{R}_{\sigma \rho}\right) \mathcal{R}^{\sigma \rho}+2 \gamma\left\{\mathcal{R}_{\mu \sigma \rho \tau} \mathcal{R}_{\nu}{ }^{\sigma \rho \tau}-2 \mathcal{R}_{\mu \sigma \nu \rho} \mathcal{R}^{\sigma \rho}\right.
\end{aligned}
$$




$$
\begin{aligned}
& \left.-2 \mathcal{R}_{\mu \sigma} \mathcal{R}_{\nu}{ }^{\sigma}+\mathcal{R} \mathcal{R}_{\mu \nu}-\frac{1}{4} g_{(5) \mu \nu}\left(\mathcal{R}_{\tau \lambda \rho \sigma} \mathcal{R}^{\tau \lambda \rho \sigma}-4 \mathcal{R}_{\sigma \rho} \mathcal{R}^{\sigma \rho}+\mathcal{R}^{2}\right)\right\} \\
& +(2 \alpha+\beta)\left(g_{(D) \mu \nu} \nabla^{2}-\nabla_{\mu} \nabla_{\nu}\right) \mathcal{R}+\beta \nabla^{2}\left(\mathcal{R}_{\mu \nu}-\frac{1}{2} g_{(D) \mu \nu} \mathcal{R}\right)=0,(2.2
\end{aligned}
$$

where $\mathcal{R}_{\sigma \nu \rho}^{\mu}$ and $\nabla_{\mu}$ are the Riemann tensor and covariant derivative for the $D$-dimensional metric $g_{(D) \mu \nu}$.

Taking $D=5$, let us carry out dimensional reduction of the field equations (2.2) relative to a constant curvature two-surface $\Sigma_{2}$, assuming the direct product ansatz

$$
\mathrm{d} s_{(5)}^{2}=g_{\alpha \beta}\left(x^{\gamma}\right) \mathrm{d} x^{\alpha} \mathrm{d} x^{\beta}+a^{2}\left(\mathrm{~d} \theta^{2}+s_{k}^{2} \mathrm{~d} \varphi^{2}\right),
$$

where $\alpha, \beta, \gamma=1,2,3$, and $k=1,0$ or -1 , with

$$
s_{1}=\sin \theta, \quad s_{0}=\theta, \quad s_{-1}=\sinh \theta
$$

$(\theta \in[0, \pi]$ for $k=1$ and $\theta \in[0, \infty]$ for $k=0,-1)$. The non-vanishing components of the Riemann tensor are

$$
\begin{aligned}
& \mathcal{R}_{\beta \gamma \delta}^{\alpha}=\delta_{\gamma}^{\alpha} R_{\beta \delta}-\delta_{\delta}^{\alpha} R_{\beta \gamma}+g_{\beta \delta} R_{\gamma}^{\alpha}-g_{\beta \gamma} R_{\delta}^{\alpha}-\frac{1}{2}\left(\delta_{\gamma}^{\alpha} g_{\beta \delta}-\delta_{\delta}^{\alpha} g_{\beta \gamma}\right) R \\
& \mathcal{R}_{\theta \varphi \theta}^{\varphi}=k, \quad \mathcal{R}^{\theta}{ }_{\varphi \theta \varphi}=k s_{k}^{2}
\end{aligned}
$$

where $R_{\alpha \beta}$ is the three-dimensional Ricci tensor. The five-dimensional and three-dimensional Ricci scalars are related by $\mathcal{R}=R+2 k a^{-2}$. The only non-vanishing components of the Lanczos tensor (the $2 \gamma$ term in (2.2) ) are those of the Einstein tensor:

$$
L_{\alpha \beta}=2 k a^{-2}\left(R_{\alpha \beta}-\frac{1}{2} R g_{\alpha \beta}\right) .
$$

Therefore, the five-dimensional Lovelock-Gauss-Bonnet equations $(\alpha=\beta=$ 0 in $(2.2)$ ) reduce to the three-dimensional cosmological Einstein equations with renormalized coupling constants.

The $(\alpha \beta)$ components of the full equations (2.2) reduce to

$$
\begin{aligned}
& \frac{\beta}{2} K_{\alpha \beta}+\left(2 \alpha+\frac{3 \beta}{4}\right)\left(g_{\alpha \beta} D^{2}-D_{\alpha} D_{\beta}\right) R \\
& +\left[\frac{1}{\kappa}+\frac{4 k(\alpha+\gamma)}{a^{2}}+\left(2 \alpha-\frac{\beta}{4}\right) R\right]\left(R_{\alpha \beta}-\frac{1}{2} g_{\alpha \beta} R\right) \\
& +\left[\frac{\Lambda-k a^{-2}}{\kappa}-\frac{2 k^{2} \alpha}{a^{4}}+\left(\frac{\alpha}{2}+\frac{11 \beta}{16}\right) R^{2}\right] g_{\alpha \beta}=0,
\end{aligned}
$$


where

$$
\begin{aligned}
K_{\alpha \beta}= & 2 D^{2} R_{\alpha \beta}-\frac{1}{2}\left(D_{\alpha} D_{\beta}+g_{\alpha \beta} D^{2}\right) R-8 R_{\alpha}{ }^{\gamma} R_{\beta \gamma} \\
& +\frac{9}{2} R R_{\alpha \beta}+\left(3 R^{\gamma \delta} R_{\gamma \delta}-\frac{13}{8} R^{2}\right) g_{\alpha \beta},
\end{aligned}
$$

and $D_{\alpha}$ is the three-dimensional covariant derivative. The solutions of the three-dimensional field equations (2.7) are restricted by the constraint, resulting from the $\theta \theta$ equation (2.2)

$$
\left(2 \alpha+\frac{\beta}{2}\right) D^{2} R+\left(\frac{\Lambda}{\kappa}+\frac{k^{2}(2 \alpha+\beta)}{a^{4}}\right)-\frac{1}{2 \kappa} R-\frac{\alpha}{2} R^{2}-\frac{\beta}{2} R^{\gamma \delta} R_{\gamma \delta}=0 .
$$

Let us make the further assumption $R=$ constant. In this case, the field equations (2.7) reduce to the equations of new massive gravity

$$
R_{\alpha \beta}-\frac{1}{2} g_{\alpha \beta} R+\lambda g_{\alpha \beta}-\frac{1}{2 m^{2}} K_{\alpha \beta}=0,
$$

with the additional constraint

$$
\frac{\beta}{2} R^{\gamma \delta} R_{\gamma \delta}+\frac{\alpha}{2} R^{2}+\frac{1}{2 \kappa} R-\left(\frac{k^{2}(2 \alpha+\beta)}{a^{4}}+\frac{\Lambda}{\kappa}\right)=0 .
$$

The parameters of the effective three-dimensional theory (2.10) are related to the parameters of the original five-dimensional theory, the compactification scale $a^{2}$ and the three-dimensional Ricci scalar by

$$
\begin{aligned}
\lambda & =c^{-1}\left[\frac{\Lambda-k a^{-2}}{\kappa}-\frac{2 k^{2} \alpha}{a^{4}}+\left(\frac{\alpha}{2}+\frac{11 \beta}{16}\right) R^{2}\right], \\
m^{-2} & =-c^{-1} \beta, \quad c=\frac{1}{\kappa}+\frac{4 k(\alpha+\gamma)}{a^{2}}+\left(2 \alpha-\frac{\beta}{4}\right) R
\end{aligned}
$$

(the real constant $m^{2}$ is not necessarily positive). When $\beta=0$ the reduced field equations (2.10) reduce to the three-dimensional cosmological Einstein equations, so it is the five-dimensional quadratic Ricci coupling $\beta \mathcal{R}^{\mu \nu} R_{\mu \nu}$ which is responsible for the occurrence of the three-dimensional quadratic couplings in (2.10).

Tracing the NMG field equations (2.10) yields the equation

$$
-\frac{1}{2} R+3 \lambda-\frac{1}{2 m^{2}}\left(R^{\gamma \delta} R_{\gamma \delta}-\frac{3}{8} R^{2}\right)=0,
$$


which can be rewritten as

$$
\frac{\beta}{2} R^{\gamma \delta} R_{\gamma \delta}-\frac{3 \beta}{16} R^{2}-\frac{c}{2} R+3 c \lambda=0 .
$$

This can be combined with (2.11) to eliminate the Ricci square term. Taking (2.12) into account, we finally arrive at the quadratic constraint on the Ricci scalar, equivalent to (2.11),

$$
2 \beta \bar{R}^{2}-[1+2(\alpha+\gamma) x] \bar{R}+4 \bar{\Lambda}-3 x-(4 \alpha-\beta) x^{2}=0,
$$

where we have put

$$
\bar{R}=\kappa R, \quad \bar{\Lambda}=\kappa \Lambda, \quad x=k \kappa a^{-2} .
$$

The special case of solutions with $k=0$ admits a simple four-dimensional interpretation. In this case the constant curvature two-surface $\Sigma_{2}$ is simply $T^{2}$, so that the five-dimensional metric can be trivially reduced to four dimensions by

$$
\mathrm{d} s_{(5)}^{2}=\mathrm{d} s_{(4)}^{2}+\mathrm{d} y^{2}
$$

with

$$
\mathrm{d} s_{(4)}^{2}=g_{\alpha \beta}\left(x^{\gamma}\right) \mathrm{d} x^{\alpha} \mathrm{d} x^{\beta}+\mathrm{d} z^{2} .
$$

So solutions of Eqs. (2.10) and (2.11) with $k=0$ (if they exist) will lead to cylindrical solutions of four-dimensional quadratic gravity. Because the Gauss-Bonnet term is a topological invariant in four dimensions, we do not expect the existence of such solutions to depend on the value of the GaussBonnet coupling constant $\gamma$.

\section{Solutions}

A number of exact solutions to the equations (2.10) of NMG are known [21, 23, 24, 25, 26, 27, 28]. Several of these have a constant Ricci scalar. In the following we shall concentrate on solutions of NMG with constant Ricci scalar, leading to non-asymptotically flat black ring solutions of five(or possibly four-) dimensional quadratic gravity.

\section{$3.1 \quad$ BTZ}

Equations (2.10) are trivially solved by Einstein metrics, leading in the case of a negative Ricci scalar to BTZ black holes [21, 23]. The resulting fivedimensional solutions are

$$
\mathrm{d} s_{(5)}^{2}=-N^{2} \mathrm{~d} t^{2}+\frac{\mathrm{d} r^{2}}{N^{2}}+r^{2}\left(\mathrm{~d} z+N^{z} \mathrm{~d} t\right)^{2}+a^{2}\left(\mathrm{~d} \theta^{2}+s_{k}^{2} \mathrm{~d} \varphi^{2}\right)
$$


where

$$
N^{2}=\frac{r^{2}}{l^{2}}-M+\frac{J^{2}}{4 r^{2}}, \quad N^{z}=\frac{J}{2 r^{2}} .
$$

These solutions depend on two dynamical parameters $M$ and $J$ (integration constants), and two scale parameters $a^{2}\left(\Sigma_{2}\right.$ scale) and $l^{2}\left(A d S_{3}\right.$ scale). The $A d S_{3}$ scale is related to the NMG parameters and to the Ricci scalar by [21, 23]

$$
R=-6 l^{-2}, \quad l^{-2}=2 m^{2}\left[-1 \pm \sqrt{1-\lambda / m^{2}}\right] .
$$

Inserting these in (2.12) and (2.15), we obtain a system of two quadratic equations for the rescaled parameters

$$
x=k \kappa a^{-2}, \quad y=\kappa l^{-2}
$$

in terms of the input parameters $\alpha, \beta, \gamma$ and $\bar{\Lambda}$ :

$$
\begin{aligned}
2 \alpha x^{2}-4(\alpha+\gamma) x y-2(3 \alpha+13 \beta) y^{2}+x-y-\bar{\Lambda} & =0 \\
(4 \alpha-\beta) x^{2}-12(\alpha+\gamma) x y-72 \beta y^{2}+3 x-6 y-4 \bar{\Lambda} & =0
\end{aligned}
$$

Eliminating $x$ between these equations generically yields an equation of the sixth degree for the variable $y$. We consider here only some special cases:

a) $\alpha=\beta=0$ (Gauss-Bonnet). In this case the equations are solved by

$$
x=\frac{2 \bar{\Lambda}}{3+4 \gamma \bar{\Lambda}}, \quad y=-\frac{\bar{\Lambda}}{3} .
$$

Assuming $\kappa>0$, and comparing the signs of $x(k)$ and $y\left(l^{-2}\right)$, we find that for $\gamma>0$, the five-dimensional geometry is $\mathrm{BTZ} \times S^{2}$ for $\bar{\Lambda}<-3 / 4 \gamma$, $\mathrm{BTZ} \times H^{2}$ for $-3 / 4 \gamma<\bar{\Lambda}<0$, and $d S_{3} \times S^{2}$ for $\bar{\Lambda}>0$, while for $\gamma<0$ it is $\mathrm{BTZ} \times H^{2}$ for $\bar{\Lambda}<0, d S_{3} \times S^{2}$ for $0<\bar{\Lambda}<-3 / 4 \gamma$, and $d S_{3} \times H^{2}$ for $\bar{\Lambda}>-3 / 4 \gamma$. For $\gamma=0$ (pure Einstein case), the solution reduces to the five-dimensional Nariai or anti-Nariai solution, with the geometry $d S_{3} \times S^{2}$ for $\bar{\Lambda}>0$, or $\mathrm{BTZ} \times H^{2}$ for $\bar{\Lambda}<0$.

b) $\bar{\Lambda}=-(2 \alpha+\beta) /(4 \alpha+\beta)^{2}$. In this case,

$$
x=-\frac{1}{4 \alpha+\beta}, \quad y=0,
$$

and the geometry is Minkowski ${ }_{3} \times S^{2}$ or Minkowski ${ }_{3} \times H^{2}$, depending on the sign of $4 \alpha+\beta$. This can be toroidally reduced to a solution of fourdimensional quadratic gravity with the geometry Minkowski ${ }_{2} \times \Sigma_{2}$. 
c) $\bar{\Lambda}=-3(3 \alpha+7 \beta) / 8(3 \alpha+4 \beta)^{2}$. This leads to

$$
x=0, \quad y=\frac{1}{3 \alpha+4 \beta},
$$

with the geometry $\mathrm{BTZ} \times T^{2}$ or $d S_{3} \times T^{2}$ according to the sign of $3 \alpha+4 \beta$. This can also be toroidally reduced to a solution of four-dimensional quadratic gravity, with the geometry $\mathrm{BTZ} \times S^{1}$ or $d S_{3} \times S^{1}$.

\subsection{AdS wave}

As shown in [24] and [25], the field equations of NMG also admit AdS wave solutions which generalize extreme BTZ black holes, and have the same constant Ricci scalar. From the stationary solutions given in [25], we obtain the solutions of five-dimensional quadratic gravity

$$
\begin{gathered}
\mathrm{d} s_{(5)}^{2}=\left[-2 l^{-2} \rho+F(\rho)\right] \mathrm{d} t^{2}-2 l F(\rho) \mathrm{d} t \mathrm{~d} z+\left[2 \rho+l^{2} F(\rho)\right] \mathrm{d} z^{2} \\
+\frac{l^{2} \mathrm{~d} \rho^{2}}{4 \rho^{2}}+a^{2}\left(\mathrm{~d} \theta^{2}+s_{k}^{2} \mathrm{~d} \varphi^{2}\right)
\end{gathered}
$$

with

$$
F(\rho)=a_{+} \rho^{p_{+}}+a_{-} \rho^{p_{-}}+M / 2, \quad p_{ \pm}=\frac{1 \pm \sqrt{m^{2} l^{2}+1 / 2}}{2},
$$

depending on three integration constants $a_{+}, a_{-}$and the BTZ mass parameter $M$. The $A d S_{3}$ scale $l^{2}$ is again given by the BTZ relation (3.3) (the sign \pm in (3.9) is independent from that in (3.3)). For $m^{2} l^{2}<-1 / 2$, the two constants $a_{ \pm}$are complex conjugate, while for $m^{2} l^{2}=-1 / 2$ and $m^{2} l^{2}=1 / 2$, the form (3.9) of $F(\rho)$ degenerates and must be replaced by forms involving logarithms, which are given in [25]. For $a_{+}=a_{-}=0$, the solution (3.8) reduces, after the coordinate transformation $\rho=r^{2} / 2$, to the extreme BTZ solution (3.1) with $J=M l$. For $a_{+}=0$ but $a_{-} \neq 0$, the solution (3.8) is asymptotic (for $\rho \rightarrow \infty$ ) to the extreme BTZ solution for $m^{2} l^{2}>1 / 2$, and weakly asymptotic (in the sense of log gravity) to extreme BTZ for $m^{2} l^{2}=1 / 2$.

The analysis of [25] shows that the three-dimensional AdS wave solutions describe regular black holes with a null Killing vector, leading to regular fivedimensional black rings, only for the discrete values $m^{2} l^{2}=17 / 2, m^{2} l^{2}=$ $7 / 2$, and $m^{2} l^{2}=1 / 2$. The black rings for $m^{2} l^{2}=17 / 2$ are actually a special case of the warped AdS black rings discussed in the next subsection, so we 
only consider the two other possibilities, focussing on the subcase $k=0$. In this case, Eq. (3.7) gives $l^{2}=\kappa(3 \alpha+4 \beta)$, leading on account of (2.12) to

$$
m^{2} l^{2}=\frac{18 \alpha-11 \beta}{2 \beta} .
$$

The massless $m^{2} l^{2}=7 / 2$ black rings are obtained for $\alpha=\beta, \bar{\Lambda}=-15 / 196 \beta$. The five-dimensional metric is given by (3.8), (3.9) with $k=0, a_{-}=M=0$, $a_{+}>0, p_{+}=3 / 2$, with a double horizon at $x \equiv \rho^{1 / 2}=0$ hiding a timelike causal singularity (for details, see [25]).

If the coupling constants are related by $\alpha=2 \beta / 3, \bar{\Lambda}=-3 / 32 \beta$, the choice $k=0$ leads to $m^{2} l^{2}=1 / 2$. The logarithmic solution which replaces (3.9) in this case leads to three possible kinds of regular five-dimensional black rings (or four-dimensional black strings). Only one of which is massive. with the four-dimensional black string metric (after an appropriate coordinate transformation)

$$
\begin{gathered}
\mathrm{d} s_{(4)}^{2}=-\frac{4 \rho^{2}}{l^{2} r^{2}} \mathrm{~d} t^{2}+r^{2}\left[\mathrm{~d} \varphi-\frac{b l \ln \left|\rho / \rho_{0}\right|}{r^{2}} \mathrm{~d} t\right]^{2}+\frac{l^{2} \mathrm{~d} \rho^{2}}{4 \rho^{2}}+\mathrm{d} z^{2} \\
\left(r^{2}=2 \rho+b l^{2} \ln \left|\rho / \rho_{0}\right|\right),
\end{gathered}
$$

depending on two parameters $b<0$ and $\rho_{0}>0$. Again, the double horizon at $\rho=0$ shields a timelike causal singularity. This spacetime is asymptotically $A d S_{3} \times S_{1}$ in the sense of log gravity, and its mass and angular momentum satisfy the extremality condition $J=M l$. That this mass, given by

$$
M=\frac{2 b \tau}{G_{3}}
$$

(with $G_{3}$ the effective three-dimensional Newton constant and $\tau$ the period of the coordinate $z$ ), is positive is non trivial. As discussed in [25, $b$ must be negative in order to avoid naked CTC, so that for a positive $G_{3}, M$ is negative. However, comparing (2.7) and (2.12), we see that the effective three-dimensional Newton constant

$$
G_{3} \propto c^{-1}=-\frac{1}{\beta m^{2}}=-\frac{\kappa}{\beta y m^{2} l^{2}}=-12 \kappa
$$

(using (3.7) with $\alpha=2 \beta / 3$ and $m^{2} l^{2}=1 / 2$ ) is negative definite, ensuring a positive mass (3.12).

The stationary AdS wave solutions of [25] are actually special cases of the more general $A d S_{3}$ wave solutions of NMG [24], which similarly lead to 
solutions of five-dimensional quadratic gravity

$$
\mathrm{d} s_{(5)}^{2}=2 l^{-2} \rho \mathrm{d} u \mathrm{~d} v+F(\rho, u) \mathrm{d} u^{2}+\frac{l^{2} \mathrm{~d} \rho^{2}}{4 \rho^{2}}+a^{2}\left(\mathrm{~d} \theta^{2}+s_{k}^{2} \mathrm{~d} \varphi^{2}\right),
$$

where $u=l z-t, v=l z+t$, and $F(\rho, u)$ is given by (3.9) with the constants $a_{ \pm}$replaced by arbitrary functions of $u$. These are different from the $A d S_{5}$ wave solutions of [5]. Another special case of AdS wave solutions of NMG is the dynamical black hole metric of [28, which can similarly be uplifted to five dimensions.

\subsection{Warped AdS}

The warped $A d S_{3}$ solutions of NMG (previously discussed as solutions of topologically massive gravity [29] (TMG) [30, 31, 32]) given in [23] lead to the following solutions of five-dimensional quadratic gravity:

$$
\begin{gathered}
\mathrm{d} s_{(5)}^{2}=-b^{2} \frac{\rho^{2}-\rho_{0}^{2}}{r^{2}} \mathrm{~d} t^{2}+r^{2}\left[\mathrm{~d} z-\frac{\rho+\left(1-b^{2}\right) \omega}{r^{2}} \mathrm{~d} t\right]^{2} \\
+\frac{1}{b^{2} \zeta^{2}} \frac{\mathrm{d} \rho^{2}}{\rho^{2}-\rho_{0}^{2}}+a^{2}\left(\mathrm{~d} \theta^{2}+s_{k}^{2} \mathrm{~d} \varphi^{2}\right),
\end{gathered}
$$

where

$$
r^{2}=\rho^{2}+2 \omega \rho+\omega^{2}\left(1-b^{2}\right)+\frac{b^{2} \rho_{0}^{2}}{1-b^{2}},
$$

and the constants $b^{2}$ and $\zeta$ are given by

$$
b^{2}=\frac{9-21 \lambda / m^{2} \mp 2 \sqrt{3\left(5+7 \lambda / m^{2}\right)}}{4\left(1-\lambda / m^{2}\right)}, \quad \zeta^{-2}=\frac{21-4 b^{2}}{8 m^{2}} .
$$

As shown in [32], these metrics correspond to regular black holes provided

$$
\zeta^{2}>0, \quad 0<b^{2}<1, \quad \omega>0 .
$$

In the limiting cases $b^{2}=1$ and $b^{2}=0$, the three-dimensional reduced metric should be replaced by the special solutions given in 32] (where $\mu_{E}$ should be replaced by $\zeta$ ).

We recall that the NMG parameters $\lambda$ and $m^{2}$ in (3.17) are related to the original five-dimensional parameters and to the three-dimensional curvature associated with (3.15),

$$
R=\frac{\zeta^{2}}{2}\left(1-4 b^{2}\right)
$$


by (2.12) and the constraint (2.15). The general case is intricate and unlightening, so we concentrate on the special case $\alpha=\gamma=0$. To compute the values of the solution parameters corresponding to a given set of values of the model parameters, we proceed in the following fashion. Eq. (3.17) can be inverted to yield, for each value of $b^{2}$, two values of the ratio $\lambda / m^{2}$ :

$$
\text { a) } \frac{\lambda}{m^{2}}=\frac{16 b^{4}-72 b^{2}+21}{\left(4 b^{2}-21\right)^{2}}, \text { b) } \frac{\lambda}{m^{2}}=1
$$

(for $\lambda / m^{2}=1$ the first equation (3.17) with the lower sign is indeterminate 1 ). From (3.19) and the second and third Eqs. (2.12), we obtain

$$
\beta \bar{R}=\frac{4 b^{2}-1}{5}, \quad \zeta^{-2}=-\frac{5}{2} \beta \kappa .
$$

Using this together with the Eqs. (2.12), we can compute another value of the ratio $\lambda / m^{2}$ in terms of the solution parameters $b^{2}$ and $x$ :

$$
\frac{\lambda}{m^{2}}=\frac{400 \beta(x-\bar{\Lambda})-11\left(4 b^{2}-1\right)^{2}}{\left(4 b^{2}-21\right)^{2}} .
$$

Comparing this with (3.20), we obtain for each value of $b^{2}$ two possible values for $\beta(x-\bar{\Lambda})$ :

$$
\text { a) } \beta(x-\bar{\Lambda})=\frac{2\left(2 b^{2}-1\right)\left(3 b^{2}-1\right)}{25}, \quad \text { b) } \beta(x-\bar{\Lambda})=\frac{48 b^{4}-64 b^{2}+113}{100} \text {. }
$$

Finally these results are combined with the constraint (2.15), written as

$$
(\beta x+1 / 2)^{2}=4 \beta(x-\bar{\Lambda})+3 / 8-2(\beta \bar{R}-1 / 4)^{2},
$$

to obtain the two possible relations between the solution parameters $x=$ $k \kappa a^{-2}$ and $b^{2}$ :

$$
\text { a) } \beta x=-\frac{1}{2} \pm \frac{\sqrt{64 b^{4}-16 b^{2}+29}}{10}, \quad \text { b) } \beta x=-\frac{1}{2} \pm \frac{\sqrt{64 b^{4}-112 b^{2}+449}}{10} \text {. }
$$

The corresponding values of the model parameter $\beta \bar{\Lambda}$ are given in terms of $b^{2}$ by (3.23) and (3.25). The sign $k$ of the two-dimensional curvature is determined from the sign of $\beta x$, taking into account that $\beta \kappa<0$ from the second equation (3.21). The outcome is that for both cases a) and b) in (3.25), $k=\mp 1$, so that there is no solution of this kind in four-dimensional

\footnotetext{
${ }^{1}$ This fact was overlooked in [23].
} 
quadratic gravity $(k=0)$. The mass of the five-dimensional warped AdS black rings is proportional to the three-dimensional mass of warped AdS black holes in NMG, which was computed in [23] to be

$$
M=\frac{\zeta^{3} b^{2}\left(1-b^{2}\right)}{2 G_{3} m^{2}} \omega \propto b^{2}\left(1-b^{2}\right) \omega,
$$

where we have used (2.12) and (3.21). This mass is positive by virtue of the regularity constraints (3.18).

\section{4 $A d S_{2} \times S^{1} \times \Sigma_{2}$}

In the special case $\lambda=m^{2}$, another solution of NMG is $A d S_{2} \times S^{1}$ [23], leading to the five-dimensional solution

$$
\mathrm{d} s^{2}=-\left(\rho^{2}-\rho_{0}^{2}\right) \mathrm{d} t^{2}-2 m^{2} \mathrm{~d} z^{2}-\frac{\mathrm{d} \rho^{2}}{2 m^{2}\left(\rho^{2}-\rho_{0}^{2}\right)}+a^{2}\left(\mathrm{~d} \theta^{2}+s_{k}^{2} \mathrm{~d} \varphi^{2}\right) .
$$

This solution - which leads to a four-dimensional solution after integrating out the cyclic coordinate $z$ - has the Minkowkian signature in the range $\rho^{2}>\rho_{0}^{2}$ provided $m^{2}<0$ and $a^{2}>0$. The three-dimensional curvature is $R=4 m^{2}$. Inserting this into the second and third equations (2.12), we obtain the linear relation

$$
1+4(\alpha+\gamma) x+4 \alpha y=0
$$

between the rescaled parameters

$$
x=\kappa k a^{-2}, \quad y=2 \kappa m^{2} .
$$

This relation shows that there is no solution of this kind for $\alpha=\gamma=0$.

One can show that there is also no solution for $\alpha=0$, even if $\gamma \neq 0$. For $\alpha \neq 0$, Eq. (3.28) can be used to eliminate $y$ from the first equation (2.12) with $\lambda=m^{2}$ and the constraint (2.15), leading to the system

$$
\begin{aligned}
& {\left[\alpha^{2}(3 \beta+4 \gamma)+2 \alpha \gamma(3 \beta+\gamma)+3 \beta \gamma^{2}\right] x^{2}+\left[\frac{\alpha}{2}(3 \beta+2 \gamma)+\frac{3 \beta \gamma}{2}\right] x} \\
& \quad+\frac{\alpha}{8}+\frac{3 \beta}{16}+\alpha^{2} \bar{\Lambda}=0 \\
& {\left[\alpha^{2}(9 \beta+8 \gamma)+4 \alpha \gamma(4 \beta+\gamma)+8 \beta \gamma^{2}\right] x^{2}+[\alpha(4 \beta+3 \gamma)+4 \beta \gamma] x} \\
& \quad+\frac{\alpha+\beta}{2}+4 \alpha^{2} \bar{\Lambda}=0
\end{aligned}
$$


which is overdetermined, meaning that there must be a specific relation between the model parameters $\alpha, \beta, \gamma$, and $\bar{\Lambda}$.

Again the general case is intricate, so we focus on the example $\gamma=0$. In this case the system (3.30) has a solution provided the model parameters are related by

$$
3 \xi^{2}+2(6 \alpha-11 \beta) \xi+12 \alpha^{2}+20 \alpha \beta+3 \beta^{2}=0,,
$$

where $\xi=16 \alpha^{2} \bar{\Lambda}$. The further assumption $\Lambda=0$ leads to the two possibilities

$$
\begin{array}{ll}
\text { a) } 2 \alpha+3 \beta=0, & y=-\frac{x}{2}=-\frac{1}{6 \beta}, \\
\text { b) } 6 \alpha+\beta=0, & y=\frac{x}{2}=\frac{1}{2 \beta} .
\end{array}
$$

Remembering that $m^{2}<0$, we find that in the first case, $k=+1$ and the five-dimensional geometry is $A d S_{2} \times S^{2} \times S^{1}$, while in the second case, $k=-1$ and the geometry is $A d S_{2} \times H^{2} \times S^{1}$.

Returning to the general case, let us point out that, contrary to appearance, these five-dimensional solutions with the geometry $A d S_{2} \times S^{1} \times \Sigma_{2}$ will not generically lead, upon integration along the $S^{1}$, to Bertotti-Robinsonlike solutions of four-dimensional quadratic gravity. The reason is that the five-dimensional equations (2.2) contain, besides the equations of fourdimensional quadratic gravity, the $(z z)$ component which leads to the additional constraint $\mathcal{L}_{4}=0$, where $\mathcal{L}_{4}$ is the integrand of the quadratic action (2.1) for $D=4$. However, as already mentioned, the case $k=0$ does lead upon toroidal reduction to a solution o four-dimensional quadratic gravity. Assuming $x=0$, we obtain from (3.28) $y=-1 / 4 \alpha$, which satisfies the system (3.30) provided

$$
\beta=0, \quad \bar{\Lambda}=-1 / 8 \alpha .
$$

Note that $\beta=0$ with $m^{2}$ finite means from (2.12) that $c=0$, contrary to the assumption made in deriving (2.10) from (2.7). However it can be checked directly that equations (2.7) are satisfied for these relations between the coupling constants if $R=-1 / 2 \kappa \alpha$. It is tempting to speculate that this $A d S_{2} \times S^{1} \times S^{1}$ solution could be the near-horizon limit of some fourdimensional black ring solution to $f(R)$ gravity. 


\section{Conclusion}

In this paper, we have constructed new solutions of five-dimensional quadratic gravity as direct products $M_{3} \times \Sigma_{2}$, where $\Sigma_{2}=S^{2}, T^{2}$ or $H^{2}$ is a constant curvature two-surface, and $M_{3}$ is a solution of three-dimensional new massive gravity with constant scalar curvature. These non-asymptotically flat solutions could represent near-horizon limits of five-dimensional asymptotically flat black strings or black rings. A number of these solutions are themselves black strings or rings (topological if $\Sigma_{2}=T^{2}$ or $H^{2}$ ) of the BTZ (3.1), null Killing vector (3.8) or warped $A d S_{3}$ (3.15) type.

A by-product of our analysis is the construction of new solutions of four-dimensional quadratic gravity with the geometry $M_{3} \times S^{1}$, obtained by toroidal reduction of the five-dimensional solutions with flat transverse space. These again include black strings or rings of the BTZ or null Killing vector type (among which the log black string (3.14)), and an $A d S_{2} \times T^{2}$ solution of $f(R)$ gravity for a specific relation between the model parameters.

We close by commenting on the relation of the present work with recent work on higher-dimensional supergravities with curvature squared invariants [33, 34, 35, 36]. In [33], a six-dimensional supergravity with quadratic couplings was reduced on a three-sphere, yielding a massive three-dimensional supergravity, which is closely related to the general massive supergravity of 26. Freezing out the scalars in this theory leads to the general massive gravity (NMG + TMG) of [21]. Similarly to what has been done here, we expect solutions of the latter theory [37, 38] to lead to Bertotti-Robinsonlike solutions of the original six-dimensional theory. The five-dimensional supergravity of [34] with a Riemann squared invariant is expected to compactify over a two-sphere to a supersymmetric extension of topologically massive gravity. Likewise, known solutions of TMG should lift to solutions of the five-dimensional theory. In [35] a higher derivative extension of a six-dimensional gauged supergravity was shown to admit solutions given by direct products $M_{4} \times \Sigma_{2}$ or $M_{3} \times \Sigma_{3}$, with $M_{p}$ and $\Sigma_{q}$ both constant curvature. In 36] a supersymmetric completion of the Einstein-Gauss-Bonnet

theory was similarly shown to admit solutions with $A d S_{3} \times S^{2}$ and $A d S_{2} \times S^{3}$ structures. Presumably these theories should also admit more general product solutions similar to those presented here.

\section{Acknowledgment}

I wish to thank Dmitry Gal'tsov for stimulating discussions. 


\section{References}

[1] K.S. Stelle, Phys. Rev. D 16 (1977) 953; Gen. Rel. Grav. 9 (1978) 353

[2] B. Zwiebach, Phys. Lett. B 156 (1985) 315

[3] H. Lü and C.N. Pope, Phys. Rev. Lett. 106 (2011) 181302 arXiv:1101.1971.

[4] S. Deser, H. Liu, H. Lü, C.N. Pope, T.C. Sisman and B. Tekin, Phys. Rev. D 83 (2011) 061502 arXiv:1101.4009.

[5] I. Gullu, M. Gurses, T.C. Sisman and B. Tekin, Phys. Rev. D 83 (2011) 084015 arXiv:1102.1921.

[6] T. Malek and V. Pravda, Phys. Rev. D 84 (2011) 024047 arXiv:1106.0331

[7] T. Malek, "Exact solutions of general relativity and quadratic gravity in arbitrary dimension", Doctoral thesis (Prague 2012) arXiv:1204.0291]

[8] H. Nariai, Sci. Rep. Tokohu Univ. 34 (1950) 160; Sci. Rep. Tokohu Univ. 35 (1951) 62

[9] P. Ginsparg and M.J. Perry, Nucl. Phys. B222 (1983) 245

[10] N. Dadhich, "On product space-time with 2-sphere of constant curvature" arXiv:gr-qc/0003026

[11] B. Bertotti, Phys. Rev. 116 (1959) 1331; I. Robinson, Bull. Acad. Polon. Sci., Ser. Math. Astr. Phys. 7 (1959) 351

[12] O.J.C. Dias and J.P. Lemos, Phys. Rev. D 68 (2003) 104010 arXiv:hep-th/0306194

[13] M. Caldarelli, L. Vanzo and Z. Zerbini, "The extremal limit of Ddimensional black holes", in "Geometrical aspects of quantum fields", A.A. Bytsenko, A.E. Goncalves and B.M. Pimentel, eds., World Scientific (Singapore 2001) arXiv:hep-th/0008136

[14] V. Cardoso, O.J.C. Dias and J.P. Lemos, Phys. Rev. D 70 (2004) 024002 arXiv:hep-th/0401192

[15] P. Figueras, H.K. Kunduri, J. Lucietti and M. Rangamani, Phys. Rev. D78 (2008) 044042 arXiv:0803.2998 
[16] H.K. Kunduri and J. Lucietti, J. Math. Phys. 50 (2009) 082502 arXiv:0806.2051]; Commun. Math. Phys. 303 (2011) 31 arXiv:1002.4656

[17] H.K. Kunduri and J. Lucietti, JHEP 0712 (2007) 015 [arXiv:0708.3695]; Living Rev. Relativity 16 (2013) 8 [arXiv:1306.2517]

[18] N. Dadhich and J.M. Pons, J. Math. Phys. 54 (2013) 102501 arXiv:1210.1109

[19] M. Gürses, Phys. Rev. D 46 (1992) 2522; M. Gürses and E. Sermutlu, Class. Quantum Grav. 12 (1995) 2799 arXiv:hep-th/9509076

[20] A. Bouchareb, C.M. Chen, G. Clément and D.V. Gal'tsov, Phys. Rev. D 88 (2013) 084048 [arXiv:1308.6461]

[21] E.A. Bergshoeff, O. Hohm and P.K. Townsend, Phys. Rev. Lett. 102 (2009) 201301 arXiv:0901.1766.

[22] S. Deser and B. Tekin, Phys. Rev. D 67 (2003) 084009 arXiv:hep-th/0212292

[23] G. Clément, Class. Quantum Grav. 26 (2009) 105015 arXiv:0902.4634

[24] E. Ayón-Beato, G. Giribet and M. Hassaïne, JHEP 0905 (2009) 029 arXiv:0904.0668

[25] G. Clément, Class. Quantum Grav. 26 (2009) 165002 arXiv:0905.0553

[26] E.A. Bergshoeff, O. Hohm and P.K. Townsend, Phys. Rev. D 79 (2009) 124042 arXiv:0905.1259.

[27] E. Ayón-Beato, A. Garbaz, G. Giribet and M. Hassaïne, Phys. Rev. D 80 (2009) 104029 arXiv:0909.1347.

[28] M. Flory and I. Sachs, Phys. Rev. D 88 (2013) 044034 arXiv:1304.1704]

[29] S. Deser, R. Jackiw and S. Templeton, Phys. Rev. Lett. 48 (1982) 975; Ann. Phys., NY 140 (1982) 372.

[30] A. Bouchareb and G. Clément, Class. Quantum Grav. 24 (2007) 5581 arXiv:0706.0263.

[31] D. Anninos, W. Li, M. Padi, W. Song and A. Strominger, JHEP 0903 (2009) 130 arXiv:0807.3040] 
[32] K. Ait Moussa, G. Clément, H. Guennoune and C. Leygnac, Phys. Rev. D 78 (2008) 064065 arXiv:0807.4241.

[33] H. Lü, C.N. Pope and E. Sezgin, JHEP 1010 (2010) 016 arXiv:1007.0173]

[34] E.A. Bergshoeff, J. Rosseel and E. Sezgin, Class. Quant. Grav. 28 (2011) 225016 arXiv:1107.2825.

[35] E. Bergshoeff, F. Coomans, E. Sezgin and A. Van Proeyen, JHEP 1207 (2012) 011 arXiv:1203.2975]

[36] M. Ozkan and Y. Pang, JHEP 1303 (2013) 158; erratum JHEP 1307 (2013) 152 [arXiv:1301.6622]

[37] S. Nam, J.D. Park and S.H. Yi, JHEP 1007 (2010) 058 arXiv:1005.1619

[38] E. Tonni, JHEP 1008 (2010) 070 arXiv:1006.3489. 